\title{
Diagnostic Accuracy of Mean Corpuscular Volume in Delineating Vitamin $\mathbf{B}_{12}$ Deficiency
}

\author{
Suprava Patel ${ }^{1 *}$, Puja Dhupar ${ }^{2}$ and Ashok Bhattar ${ }^{3}$ \\ ${ }^{1}$ Department of Biochemistry, All India Institute of Medical Sciences (AIIMS), Raipur, Chhattisgarh, India \\ ${ }^{2}$ Balgopal Hospital and Research Institute, Raipur, Chhattisgarh, India
}

${ }^{*}$ Corresponding author: Suprava Patel, Department of Biochemistry, All India Institute of Medical Sciences (AlIMS), Raipur, Chhattisgarh, India, Tel: +918518881707; E-mail: dr_suprava@yahoo.co.in

Received: September 15, 2017; Accepted: September 23, 2017; Published: September 29, 2017

Citation: Patel S, Dhupar P, Bhattar A (2017) Diagnostic Accuracy of Mean Corpuscular Volume in Delineating Vitamin B 12 Deficiency. Ann Clin Lab Res Vol.5:No.3:195.

\section{Abstract}

Background: Nutritional deficiency, especially $B_{12}$ deficiency is quite common in India. The manifestation can range from anemia usually megaloblastic anemia as an early indicator to neurological sequel that develops later. Evaluation of Mean Corpuscular Volume (MCV) as a screening parameter has been highly unreliable and misleading. This has resulted in delayed diagnosis with irreversible changes.

Aim: Our study aimed at evaluating the sensitivity, specificity and diagnostic accuracy of MCV in delineating vitamin $B_{12}$ deficiency.

Methods: The study population consisted of 501 adolescent girls aged 10 to 18 years going to schools in the rural areas of the district. Pre-designed proforma comprising of required demographic profile and detail history were filled-up. The cut off value used for serum $B_{12}$ was $200 \mathrm{pg} / \mathrm{ml}$ and that of MCV was $100 \mathrm{fL}$.

Findings: The prevalence of vitamin $\mathrm{B}_{12}$ deficiency in rural areas was found to be $58.58 \%$ in adolescent school going girls. The $B_{12}$ deficient population depicted significant association with vegetarian diet $(p<0.001)$ and BMI $(p=0.005)$. The sensitivity and specificity of MCV to screen out $\mathrm{B}_{12}$ deficiency were calculated to be $10.14 \%$ and $92.82 \%$ respectively. Accuracy of MCV as an indicator for $B_{12}$ deficiency was estimated to be $45.05 \%$. From all the above data, the percentage of subjects missed to be diagnosed for $B_{12}$ deficiency of all deficient subjects was calculated to be $89.86 \%$.

Conclusion: The study revealed that almost every alternate adolescent girl was found to be $B_{12}$ deficient. Raised MCV was found in only $1 \%$ cases and the sensitivity was very low. Hence, MCV should not be the screening criteria for $B_{12}$ deficiency which might be misleading.
Keywords: Adolescent girls; Vitamin $\mathrm{B}_{12}$ deficiency; Mean corpuscular volume; Diagnostic accuracy; Sensitivity

\section{Introduction}

Many studies have evidenced for high prevalence of $B_{12}$ deficiency in India. Besides malnutrition, mal-absorptive disorders due to tropical sprue, gastric atrophy, Helicobacter pylori infection are among the various etiological factors for $\mathrm{B}_{12}$ deficiency. Strict vegetarian diet is also a proven factor for low $B_{12}$ level in body [1-3]. Vitamin $B_{12}$ deficiency manifestation can range from anemia usually megaloblastic anemia as an early indicator to neurological sequel that develops later. For years, complete hemogram, particularly mean corpuscular volume (MCV) has been used as screening criteria for $B_{12}$ deficiency [4,5]. Macrocytosis, MCV $>100$ femtolitres (fl) often precedes anemia that occurs due to defect in red blood cell (RBC) maturation though hemoglobin synthesis proceeds normally $[6,7]$.

Recent published data have unraveled that MCV might not be informative in subclinical $B_{12}$ deficiency. However, very few studies have explored regarding its diagnostic accuracy in $B_{12}$ deficiency. Our study aimed at evaluating the sensitivity, specificity and diagnostic accuracy of MCV in delineating vitamin $B_{12}$ deficiency.

\section{Materials and Methods}

The study population consisted of 501 adolescent girls aged 10-18 years going to schools in the rural areas of the district. The study was approved by the Institution Ethics Committee and requisite consent was taken from parents/legally accepted representatives (LAR). Those with history of hemolytic disease were excluded in the study. Of all 496 subjects were taken up for data analysis after excluding 5 cases with very high serum $B_{12}$ values who later on gave consent for being treated with $\mathrm{B}_{12}$ supplementation.

Pre-designed proforma comprising of required demographic profile and detail history were filled-up. Blood sample were collected under all aseptic conditions and sent to laboratory 
for analysis of complete hematogram, serum iron, and vitamin $\mathrm{B}_{12}$ levels. Complete hematogram was analyzed in automated analyzer BC-5180 from Mindray.

Serum $B_{12}$ was measured in Electrochemiluminescence method in Cobas e-400 immunoassay autoanalyzer from Roche Diagnostics and serum Iron was measured by IronFerrozine method in Biosystem B 400 fully automated clinical autoanalyzer from Biosystem reagent and instruments.

The cut off value for serum $B_{12}$ was $200 \mathrm{pg} / \mathrm{ml}$, accordingly, the study population was categorized as $B_{12}$ deficient $\left(B_{12}<200\right.$ $\mathrm{pg} / \mathrm{ml})$ and $B_{12}$ non-deficient groups $\left(B_{12} \geq 200 \mathrm{pg} / \mathrm{ml}\right)[8]$. In order to evaluate the accuracy of $\mathrm{MCV}$ as a screening parameter, a value of $\mathrm{MCV}>100 \mathrm{fl}$ was considered as macrocytic [2].

SPSS 17 software was used for statistical analysis. Categorical data were compared using Chi-square analysis and binary-logistic regression analysis. Independent ' $\mathrm{t}$ ' test was used for comparison of patients' laboratory data of the groups. Sensitivity, specificity, positive and negative predictive value (PPV and NPV), accuracy and receiver operating curve (ROC) were calculated for MCV to verify its efficiency as screening parameter. Statistical significance was accepted at $p<0.05$ (Table 1).

\section{Results}

The prevalence of vitamin $B_{12}$ deficiency in rural areas was found to be $58.58 \%$ in adolescent school going girls. The percentage of anemia in $B_{12}$ deficient population was observed to be $43.1 \%(n=125 / 290)$ of which only $13(10.4 \%)$ anemic subjects documented raised MCV. The estimated blood and serum parameters, except for serum $B_{12}$ levels, neither recorded any significant differences among the two groups nor showed any significant correlation with serum $B_{12}$ values. (Table 1).

Table 1 Comparison of mean \pm SD of the estimated parameters between the two groups (Vitamin $B_{12}$ deficient and nondeficient).

\begin{tabular}{|c|c|c|c|}
\hline Parameters & $\begin{array}{c}\mathbf{B}_{12} \text { non-deficient } \\
\text { group (n=205) }\end{array}$ & $\begin{array}{c}\mathbf{B}_{12} \text { deficient } \\
\text { group (n=290) }\end{array}$ & P value \\
\hline $\mathrm{Hb}$ & $12.15 \pm 1.24$ & $12.01 \pm 1.44$ & 0.25 \\
\hline $\mathrm{MCV}$ & $87.88 \pm 11.72$ & $86.94 \pm 10.41$ & 0.35 \\
\hline $\mathrm{MCH}$ & $27.74 \pm 4.53$ & $27.73 \pm 7.33$ & 0.97 \\
\hline $\mathrm{MCHC}$ & $30.40 \pm 2.47$ & $30.46 \pm 2.21$ & 0.78 \\
\hline $\mathrm{RDW}$ & $14.38 \pm 2.20$ & $14.76 \pm 3.25$ & 0.11 \\
\hline Iron & $70.38 \pm 37.38$ & $66.77 \pm 39.47$ & 0.30 \\
\hline
\end{tabular}

The $B_{12}$ deficient population depicted significant association with vegetarian diet $(p<0.001)$ and $B M I(p=0.005)$ but not with other demographic profiles like age group, worm infestation and SES (Tables 2 and 3). As per logistic regression analysis, the odds of having $B_{12}$ deficiency was found to be 7.67 times greater for vegetarian subjects as opposed to subjects preferring mixed diet ( $\mathrm{Cl}: 4.59-12.82 ; \mathrm{p}=0.001)$.

Table 2 Association of serum $B_{12}$ values with diet pattern.

\begin{tabular}{|c|c|c|c|}
\hline Diet & Non-deficient & $\mathbf{B}_{12}$ deficient & Total \\
\hline Vegetarian & 19 & 135 & 154 \\
\hline Mixed & 186 & 155 & 341 \\
\hline Total & 205 & 290 & 495 \\
\hline
\end{tabular}

Chi-square test $p<0.001^{* *}$

Table 3 Association of serum $B_{12}$ values with $B M I$ in study population.

\begin{tabular}{|c|c|c|c|}
\hline BMI & Non deficient & B $_{12}$ deficient & Total \\
\hline$<18.5$ & 147 & 166 & 313 \\
\hline $18.5-25$ & 56 & 117 & 173 \\
\hline $25.1-29$ & 2 & 7 & 9 \\
\hline Total & 205 & 290 & 495 \\
\hline Chi square test $p=0.005^{*}$ & \\
\hline
\end{tabular}

The frequency of occurrence of raised MCV in the $B_{12}$ deficient population was $01 / 100(n=29 / 290)$. The sensitivity and specificity of $\mathrm{MCV}$ to screen out $\mathrm{B}_{12}$ deficiency were calculated to be $10.14 \%$ and $92.82 \%$ respectively. The PPV and NPV were respectively $65.91 \%$ and $43.01 \%$.

Accuracy of MCV as an indicator for $B_{12}$ deficiency was estimated to be $45.05 \%$. From all the above data, the percentage of subjects missed to be diagnosed for $B_{12}$ deficiency of all deficient subjects was calculated to be $89.86 \%$. The receiver operating curve (ROC) documented an area of 0.45 indicating MCV as an unreliable test for screening (Figure 1 and Table 4).

\section{Discussion}

The prevalence of vitamin $\mathrm{B}_{12}$ deficiency in adolescent girls was found to be $58.58 \%$ which corroborated with the result published by Rajendra et al., who depicted prevalence of $50 \%$ in adolescent groups in Meeyannoor, Kerala [9]. Similarly, Kapil et al. also reported prevalence of $68.3 \%$ in children of 12 to 18 years in New Delhi [10]. In contrary, Jain et al. in their study revealed low sero-prevalence of vitamin $B_{12}$ deficiency (36.5\%) in Jaipur [2]. The difference in prevalence could be because the study was based on retrospective data analysis from the records, hence, the age group and also the cut-off values utilized were different. Similarly, study conducted by Kapil et al. in New Delhi, documented higher prevalence than that of us $(73.5 \%)$ [11].

The reason could be attributed to diet pattern adopted by the study population. $68.95 \%$ adolescent population in our study preferred mixed diet pattern as against $46.1 \%$ in New Delhi, the reported percentage consumption of non-vegetarian diet in New Delhi as tabulated by Agrawal et al. [12]. 
Vegetarians were found to be 7.67 times more likely to have low $B_{12}$ levels than those who also prefer non-vegetarian food.

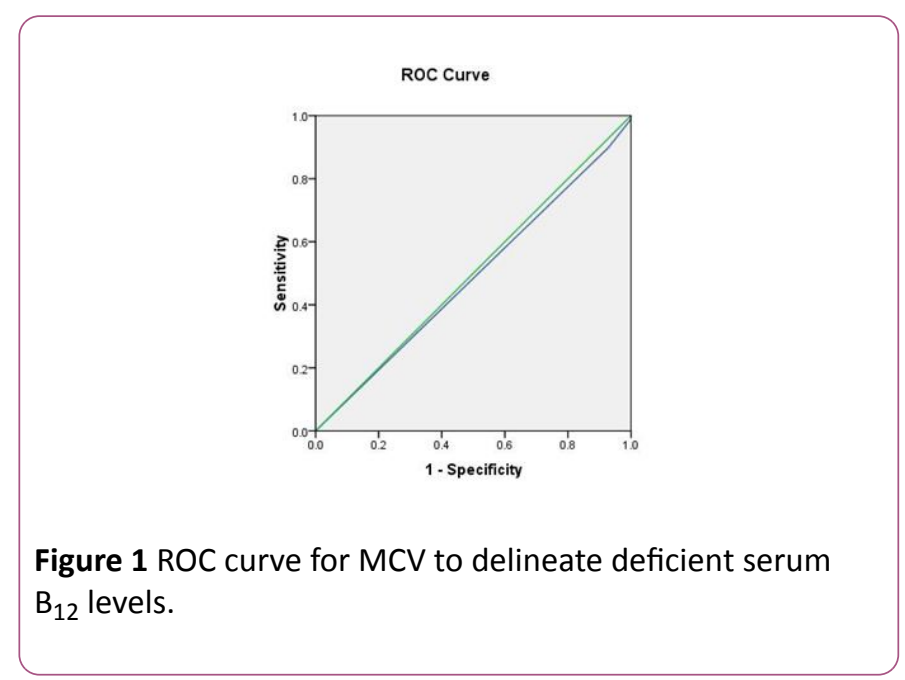

Table 4 Area under the Curve: Tests results variable(s): indicator.

\begin{tabular}{|c|c|c|c|c|}
\hline Area & \multirow{2}{*}{$\begin{array}{c}\text { Std. } \\
\text { error }\end{array}$} & $\begin{array}{c}\text { Asymptomati } \\
\text { c sig. }\end{array}$ & \multicolumn{2}{|c|}{$\begin{array}{c}\text { Asymptomatic 95\% } \\
\text { Confidence Interval }\end{array}$} \\
\cline { 4 - 5 } & & & $\begin{array}{c}\text { Lower } \\
\text { Bound }\end{array}$ & Upper Bound \\
\hline 0.485 & 0.026 & 0.563 & 0.433 & 0.536 \\
\hline
\end{tabular}

Due to lack of gold standard for diagnosis of $\mathrm{B}_{12}$ deficiency, the physicians at primary health care centers still rely on high MCV as diagnostic criterion for the same. Raised MCV was reported in only $1 \%$ of $B_{12}$ deficient population. Khanduri et al. in their study also observed elevated MCV in only 1 subject out of 50 adults and commented that MCV was not informative [13]. Jain et al. recorded $10.36 \%$ whereas Bhatia et al. published $25 \%$ macrocytosis in $B_{12}$ deficient subjects [1,2]. The difference could be because in the latter two studies were based on retrieval of laboratory data of serum $B_{12}$ assays done in their respective hospitals. Other studies also suggested that raised MCV might not necessarily accompany low serum $B_{12}$ level, hence, physicians need to be judicious enough to rule out $B_{12}$ deficiency in case of normal MCV values $[4,5,14]$.

The calculated sensitivity and specificity of MCV to screen out $\mathrm{B}_{12}$ deficiency was calculated to be $10.14 \%$ and $92.82 \%$ respectively and the diagnostic accuracy was found to be $45 \%$. It was further calculated that nearly $89 \% \mathrm{~B}_{12}$ deficient cases would have been missed on basis of sensitivity. No significant correlation was found between serum $B_{12}$ levels and $\mathrm{MCV}$ values. Also the area under ROC indicated that MCV is highly unreliable screening test. Oosterhuis et al. in their study also worked out on the diagnostic value of elevated MCV for $\mathrm{B}_{12}$ deficiency and reported sensitivity of $17 \%$ to $30 \%$. Accordingly they implicated that up to $84 \%$ of $B_{12}$ deficient cases would be missed [14]. Similarly, Thompson et al. got the finding that $82 \%$ subjects with low $B_{12}$ levels had MCV values below $95 \mathrm{fL}$ [15].

\section{Limitations}

Our study was not without any limitations. The study was based completely on serum $B_{12}$ levels. Serum folate was not measured because of fund constraint and also because iron folic acid supplementation are being provided to the adolescent girls which might influence the findings. Evaluation of other biochemical markers such as homocysteine and methylmalonic acid would have validated $B_{12}$ deficiency more accurately. However, we believe that estimation of these parameters would not be feasible in the peripheral health sector in our area because of lack of adequate instrumentation and other resources.

\section{Conclusion}

The study revealed that almost every alternate adolescent girl was found to be $B_{12}$ deficient. Raised MCV was found in only $1 \%$ cases and the sensitivity was very low. Hence, MCV should not be the screening criteria for $B_{12}$ deficiency which might be misleading.

\section{Acknowledgements}

The authors acknowledge the immense contribution of all laboratory professionals of Balgopal Children Hospital and Research Center and Department of Biochemistry, All India Institute of Medical Sciences, Raipur, Chhattisgarh in accomplishing this work.

\section{Funding Source}

This study was not funded by any pharmaceutical company or any other funding agency either for writing the manuscript or for submission for publication.

\section{Conflict of Interests}

The authors have no competing interests

\section{References}

1. Bhatia P, Kulkarni JD, Pai SA (2012) Vitamin $B_{12}$ deficiency in India: Mean corpuscular volume is an unreliable screening parameter. The Nat Med J Ind 25: 336-338.

2. Jain R, Kapil M, Gupta GN (2012) M.C.V. should not be the only criteria to order vitamin $B_{12}$ for anemia under evaluation. Open J Gastroenterol 2: 187-190.

3. Allen LH (2008) Causes of vitamin $B_{12}$ and folate deficiency Food Nutr Bull 29: S20-S34

4. Den Elzen WP, Van Der Weele GM, Gussekloo J, Westendorp RG, Assendelft WJ (2010) Subnormal vitamin $B_{12}$ concentrations and anaemia in older people: A systematic review. BMC Geriatr 10: 42.

5. Gupta AK, Damji A, Uppaluri A (2004) Vitamin $B_{12}$ deficiency. Prevalence among South Asians at a Toronto clinic. Can Fam Physician Med Fam Can. 50: 743-747. 
6. Veda P (2013) Evaluation of macrocytosis in routine hemograms Indian J Hematol Blood Transfus 29: 26-30.

7. Rajashekar R, Patel S, Kulkarni P (2017) Discriminant functions in the diagnosis of vitamin B12 deficiency anemia, the value of RDW-SD: An analytical study. NJLM 6: 1-6.

8. Allen LH (2009) How common is vitamin $B_{12}$ deficiency? Am J Clin Nutr 89: 693-696.

9. Rajendra R, Sudha S, Sreekanthan S, Vijayakumar A, Rajendran $R$, et al. (2014) Iron, vitamin $B_{12}$ and folate deficiency in adolescents having nutritional anaemia. J Evol Med Dent Sci 3: 10626-10633.

10. Kapil U, Sareen N (2014) Prevalence of ferritin, folate and vitamin $B_{12}$ deficiencies amongst children in 5-18 years of age in Delhi. Indian J Pediatr 81: 312-312.

11. Kapil U, Bhadoria AS (2014) Prevalence of folate, ferritin and cobalamin deficiencies amongst adolescent in India. J Fam Med Prim Care. 3: 247-249.
12. Agrawal S, Millett CJ, Dhillon PK, Subramanian SV, Ebrahim S (2014) Type of vegetarian diet, obesity and diabetes in adult Indian population. Nutr J 13: 89.

13. Khanduri U, Sharma A, Joshi A (2005) Occult cobalamin and folate deficiency in Indians. Nat Med J India 18: 182-183.

14. Oosterhuis WP, Niessen RW, Bossuyt PM, Sanders GT, Sturk A (2000) Diagnostic value of the mean corpuscular volume in the detection of vitamin $B_{12}$ deficiency. Scand J Clin Lab Invest 60: 9-18.

15. Thompson WG, Babitz L, Cassino C, Freedman M, Lipkin M (1987) Evaluation of current criteria used to measure vitamin $B_{12}$ levels. Am J Med 82: 291-294. 